\title{
Steiner 3-diameter, maximum degree and size of a graph*
}

\author{
Yaping $\mathrm{Mao}^{\dagger}$ \\ School of Mathematics and Statistics, Qinghai Normal \\ University, Xining, Qinghai 810008, China
}

\begin{abstract}
The Steiner $k$-diameter $\operatorname{sdiam}_{k}(G)$ of a graph $G$, introduced by Chartrand, Oellermann, Tian and Zou in 1989, is a natural generalization of the concept of classical diameter. When $k=2, \operatorname{sdiam}_{2}(G)=\operatorname{diam}(G)$ is the classical diameter. The problem of determining the minimum size of a graph of order $n$ whose diameter is at most $d$ and whose maximum is $\ell$ was first introduced by Erdös and Rényi. In this paper, we generalize the above problem for Steiner $k$-diameter, and study the problem of determining the minimum size of a graph of order $n$ whose Steiner 3 -diameter is at most $d$ and whose maximum degree is at most $\ell$.
\end{abstract}

Keywords: Diameter; Steiner diameter; maximum degree.

AMS subject classification 2010: 05C05; 05C12; 05C35.

\section{Introduction}

All graphs in this paper are undirected, finite and simple. We refer to [7] for graph theoretical notation and terminology not described here. For a graph $G$, let $V(G), E(G)$ and $e(G)$ denote the set of vertices, the set of edges and the size of $G$, respectively. We divide our introduction into the following four subsections to state the motivations and our results of this paper.

\footnotetext{
*Supported by the National Science Foundation of China (Nos. 11551001, 11161037, 11101232, 11461054) and the Science Found of Qinghai Province (No. 2014-ZJ-907).

${ }^{\dagger}$ E-mail: maoyaping@ymail.com
} 


\subsection{Distance and its generalizations}

Distance is one of the most basic concepts of graph-theoretic subjects. If $G$ is a connected graph and $u, v \in V(G)$, then the distance $d(u, v)$ between $u$ and $v$ is the length of a shortest path connecting $u$ and $v$. If $v$ is a vertex of a connected graph $G$, then the eccentricity $e(v)$ of $v$ is defined by $e(v)=\max \{d(u, v) \mid u \in V(G)\}$. Furthermore, the radius $\operatorname{rad}(G)$ and diameter $\operatorname{diam}(G)$ of $G$ are defined by $\operatorname{rad}(G)=\min \{e(v) \mid v \in V(G)\}$ and $\operatorname{diam}(G)=\max \{e(v) \mid v \in V(G)\}$. These last two concepts are related by the inequalities $\operatorname{rad}(G) \leq \operatorname{diam}(G) \leq 2 \operatorname{rad}(G)$. The center $C(G)$ of a connected graph $G$ is the subgraph induced by the vertices $u$ of $G$ with $e(u)=\operatorname{rad}(G)$. Recently, Goddard and Oellermann gave a survey paper on this subject, see [29].

The distance between two vertices $u$ and $v$ in a connected graph $G$ also equals the minimum size of a connected subgraph of $G$ containing both $u$ and $v$. This observation suggests a generalization of distance. The Steiner distance of a graph, introduced by Chartrand, Oellermann, Tian and Zou in 1989, is a natural and nice generalization of the concept of classical graph distance. For a graph $G(V, E)$ and a set $S \subseteq V(G)$ of at least two vertices, an $S$-Steiner tree or a Steiner tree connecting $S$ (or simply, an S-tree) is a such subgraph $T\left(V^{\prime}, E^{\prime}\right)$ of $G$ that is a tree with $S \subseteq V^{\prime}$. Let $G$ be a connected graph of order at least 2 and let $S$ be a nonempty set of vertices of $G$. Then the Steiner distance $d_{G}(S)$ among the vertices of $S$ (or simply the distance of $S$ ) is the minimum size among all connected subgraphs whose vertex sets contain $S$. Note that if $H$ is a connected subgraph of $G$ such that $S \subseteq V(H)$ and $|E(H)|=d_{G}(S)$, then $H$ is a tree. Observe that $d_{G}(S)=\min \{e(T) \mid S \subseteq V(T)\}$, where $T$ is subtree of $G$. Furthermore, if $S=\{u, v\}$, then $d_{G}(S)=d(u, v)$ is nothing new but the classical distance between $u$ and $v$. Set $d_{G}(S)=\infty$ when there is no $S$-Steiner tree in $G$.

The distance between two vertices $u$ and $v$ in a connected graph $G$ also equals the minimum size of a connected subgraph of $G$ containing both $u$ and $v$. This observation suggests a generalization of distance. The Steiner distance of a graph, introduced by Chartrand, Oellermann, Tian and Zou in 1989, is a natural and nice generalization of the concept of classical graph distance. For a graph $G(V, E)$ and a set $S \subseteq V(G)$ of at least two vertices, an $S$-Steiner tree or a Steiner tree connecting $S$ (or simply, an S-tree) is a such subgraph $T\left(V^{\prime}, E^{\prime}\right)$ of $G$ that is a tree with $S \subseteq V^{\prime}$. Let $G$ be a connected graph of order at least 2 and let $S$ be a nonempty set of vertices of $G$. Then the Steiner distance $d(S)$ among the vertices of $S$ (or simply the distance of $S$ ) is the minimum size among all connected subgraphs whose vertex sets contain $S$. Note that if $H$ is a connected subgraph of $G$ such that $S \subseteq V(H)$ and $|E(H)|=d(S)$, then $H$ is a tree. Observe that $d_{G}(S)=\min \{e(T) \mid S \subseteq V(T)\}$, where $T$ is subtree of $G$. Furthermore, if $S=\{u, v\}$, then $d_{G}(S)=d(u, v)$ is nothing new but the classical distance between $u$ and $v$. Set $d_{G}(S)=\infty$ 
when there is no $S$-Steiner tree in $G$.

Observation 1.1 Let $G$ be a graph of order $n$ and $k$ be an integer with $2 \leq k \leq n$. If $S \subseteq V(G)$ and $|S|=k$, then $d_{G}(S) \geq k-1$.

Let $n$ and $k$ be two integers with $2 \leq k \leq n$. The Steiner $k$-eccentricity $e_{k}(v)$ of a vertex $v$ of $G$ is defined by $e_{k}(v)=\max \{d(S)|S \subseteq V(G)| S \mid,=k$, and $v \in S\}$. The Steiner $k$-radius of $G$ is $\operatorname{srad}_{k}(G)=\min \left\{e_{k}(v) \mid v \in V(G)\right\}$, while the Steiner $k$-diameter of $G$ is $\operatorname{sidam}_{k}(G)=\max \left\{e_{k}(v) \mid v \in V(G)\right\}$. Note for every connected graph $G$ that $e_{2}(v)=e(v)$ for all vertices $v$ of $G$ and that $\operatorname{srad}_{2}(G)=\operatorname{rad}(G)$ and $\operatorname{sdiam}_{2}(G)=\operatorname{diam}(G)$.

Let $G$ be a $k$-connected graph and $u, v$ be a pair of vertices of $G$. Let $P_{k}(u, v)=$ $\left\{P_{1}, P_{2}, \cdots, P_{k}\right\}$ be a family of $k$ internally vertex-disjoint paths between $u$ and $v$ and $l\left(P_{k}(u, v)\right)$ be the length of the longest path in $P_{k}(u, v)$. Then the $k$-distance $d_{k}(u, v)$ between vertices $u$ and $v$ is the smallest $l\left(P_{k}(u, v)\right)$ among all $P_{k}(u, v)$ 's and the $k$-diameter $d_{k}(G)$ of $G$ is the maximum $k$-distance $d_{k}(u, v)$ over all pairs $u, v$ of vertices of $G$. The concept of $k$-diameter has its origin in the analysis of routings in networks as described by Chung [12, Du, Lyuu and Hsu [16], Hsu [34, 35], Meyer and Pradhan [42].

\subsection{Application backgrounds}

Perhaps the most famous Steiner type problem is the Steiner tree problem. The original Steiner tree problem was stated for the Euclidean plane: Given a set of points on the plane, the goal is to connect these points, and possibly additional points, by line segments between some pairs of these points such that the total length of these line segments is minimized. The graph theoretical version [30, 36] is as follows: Given a graph and a set of vertices $S$, find a connected subgraph with minimum number of edges that contains $S$. This is, in general, an NP-hard problem [33]. There is also a corresponding weighted version. Obviously, this has applications in computer science and electrical engineering. For example, a graph can be a computer network with vertices being computers and edges being links between them. Here the Steiner tree problem is to find a subnetwork containing these computers with the least number of links. We can replace processors by electrical stations for applications in electrical networks.

Li et al. 24] gave such a concept. They defined the $k$-center Steiner Wiener index $S W_{k}(G)$ of the graph $G$ to be

$$
S W_{k}(G)=\sum_{\substack{S \subseteq V(G) \\|S|=k}} d(S) .
$$

For $k=2$, it coincides with the ordinary Wiener index. One usually considers $S W_{k}$ for $2 \leq$ $k \leq n-1$. However, the above definition can be extended to $k=1$ and $k=n$ as well where 
$S W_{1}(G)=0$ and $S W_{n}(G)=n-1$. There are other related concepts such as the Steiner Harary index. Both indices have chemical applications [20, 23]. In addition, the Steiner degree distance by Gutman [22], Steiner Harary index by Furtula, Gutman, Katanić [20], Steiner Gutman index by Mao and Das [39], Steiner hyper-Wiener index by Tratnik [44] was introduced and studied. We refer the readers to [20, 23, 22, 24, 24, 39, 40, 41] for details.

\subsection{Recent progress of Steiner distance}

In [14, Dankelmann, Swart and Oellermann obtained a bound on $\operatorname{sdiam}_{k}(G)$ for a graph $G$ in terms of the order of $G$ and the minimum degree of $G$, that is, $\operatorname{sdiam}_{k}(G) \leq$

$\frac{3 p}{\delta+1}+3 n$. Later, Ali, Dankelmann, Mukwembi 2] improved the bound of $\operatorname{sdiam}_{k}(G)$ and showed that $\operatorname{sdiam}_{k}(G) \leq \frac{3 p}{\delta+1}+2 n-5$ for all connected graphs $G$. Moreover, they constructed graphs to show that the bounds are asymptotically best possible.

Arunandhi, Cheng and Melekian [3] studied the Steiner $k$-diameters of the tensor product of complete graphs.

The following observation is easily seen.

Observation 1.2 Let $k$ be an integer with $2 \leq k \leq n$.

(1) If $H$ is a spanning subgraph of $G$, then $\operatorname{sdiam}_{k}(G) \leq \operatorname{sdiam}_{k}(H)$.

(2) For a connected graph $G, \operatorname{sdiam}_{k}(G) \leq \operatorname{sdiam}_{k+1}(G)$.

In [10], Chartrand, Okamoto, Zhang obtained the following results.

Theorem 1.1 [10] Let $G$ be a connected graph of order $n$. Then

$$
k-1 \leq \operatorname{sidm}_{k}(G) \leq n-1 .
$$

Moreover, the bounds are sharp.

\subsection{Classical extremal problem and our generalization}

What is the minimal size of a graph of order $n$ and diameter $d$ ? What is the maximal size of a graph of order $n$ and diameter $d$ ? It is not surprising that these questions can be answered without the slightest effort (see [5]) just as the similar questions concerning the connectivity or the chromatic number of a graph. The class of maximal graphs of order $n$ and diameter $d$ is easy to describe and reduce every question concerning maximal graphs to a not necessarily easy question about binomial coefficient, as in [31, 32, 43, 45]. Therefore, the authors study the minimal size of a graph of order $n$ and under various additional conditions. 
Erdös and Rényi [18] introduced the following problem. Let $d, \ell$ and $n$ be natural numbers, $d<n$ and $\ell<n$. Denote by $\mathscr{H}(n, \ell, d)$ the set of all graphs of order $n$ with maximum degree $\ell$ and diameter at most $d$. Put

$$
e(n, \ell, d)=\min \{e(G): G \in \mathscr{H}(n, \ell, d)\} .
$$

If $\mathscr{H}(n, \ell, d)$ is empty, then, following the usual convention, we shall write $e(n, \ell, d)=\infty$. For more details on this problem, we refer to [5, 6, 18, 19].

We now consider the generalization of the above problem. Let $d, \ell$ and $n$ be natural numbers, $d<n$ and $\ell<n$. Denote by $\mathscr{H}_{k}(n, \ell, d)$ the set of all graphs of order $n$ with maximum degree $\ell$ and $\operatorname{sdiam}_{k}(G) \leq d$. Put

$$
e_{k}(n, \ell, d)=\min \left\{e(G): G \in \mathscr{H}_{k}(n, \ell, d)\right\} .
$$

If $\mathscr{H}_{k}(n, \ell, d)$ is empty, then, following the usual convention, we shall write $e_{k}(n, \ell, d)=\infty$. From Theorem [1.1, we have $k-1 \leq d \leq n-1$.

In this paper, we focus our attention on the case $k=3$, and study the exact value of $e_{3}(n, \ell, d)$ for $d=n-1, n-2, n-3,2,3$. For general $d$, we give an upper bound of $e_{3}(n, \ell, d)$.

\section{Preliminaries}

The following observation is immediate.

Observation 2.1 [37] (1) For a cycle $C_{n}$, $\operatorname{sdiam}_{k}\left(C_{n}\right)=\left\lfloor\frac{n(k-1)}{k}\right\rfloor$;

(2) For a complete graph $K_{n}$, $\operatorname{siam}_{k}\left(K_{n}\right)=k-1$.

The following result can be easily proved, which will be used later.

Theorem 2.1 For $2 \leq \ell \leq n-1$ and $3 \leq k \leq n$,

$$
e_{k}(n, \ell, n-1)=n-1 \text {. }
$$

Proof. For $\ell=2$, let $G$ be a path of order $n$. Since $\operatorname{sdiam}_{k}(G) \leq n-1, \Delta(G)=2$ and $e(G)=n-1$, it follows that $e_{k}(n, \ell, n-1) \leq n-1$. On the other hand, since we only consider connected graphs, it follows that $e(G) \geq n-1$ for a connected graph $G$ of order $n$. So $e_{k}(n, \ell, n-1)=n-1$, as desired.

Suppose $3 \leq \ell \leq n-1$. Let $G$ be a graph obtained from a star $S_{\ell}$ and a path $P_{n-\ell+1}$ by identifying the center of the star and one endpoint of the path. Since $\Delta(G)=\ell$, $\operatorname{sdiam}_{k}(G) \leq n-1$ and $e(G)=n-1$, it follows that $e_{k}(n, \ell, n-1) \leq n-1$. On the other hand, since we only consider the connected graph, it follows that $e(G) \geq n-1$ for a connected graph $G$ is of order $n$. So $e_{k}(n, \ell, n-1)=n-1$. The proof is now complete. 
Lemma 2.1 Let $T$ be a tree of order $n$ with $r$ leaves in $T$. If $r \geq 4$, then $\operatorname{sdiam}_{3}(T) \leq$ $n-r+2$.

Proof. Let $v_{1}, v_{2}, \cdots, v_{r}$ be all the leaves of $T$. For any $S \subseteq V(T)$ and $|S|=3$, there are at least $r-3$ leaves in $T$ not belonging to $S$. Pick up $r-3$ of these vertices of degree 1 and then delete them. The resulting graph is also a tree of order $n-(r-3)=n-r+3$, say $T^{\prime}$. By our choosing, it is clear that $S \subseteq V\left(T^{\prime}\right)$, that is, the tree $T^{\prime}$ is an $S$-Steiner tree. Therefore, $d_{G}(S) \leq e\left(T^{\prime}\right)=n-r+3-1=n-r+2$. From the arbitrariness of $S$, we have $\operatorname{sdiam}_{3}(T) \leq n-r+2$. The proof is now complete.

Lemma 2.2 Let $n, d$, $\ell$ be three integers with $2 \leq d \leq n-2$ and $n-d+2 \leq \ell \leq n-2$. Then

$$
e_{3}(n, \ell, d)=n-1
$$

Proof. Let $G$ be a graph obtained from a star $S_{\ell}$ and a path $P_{n-\ell+1}$ by identifying the center of the star and one end of the path. Clearly, $\Delta(G)=\ell$ and $G$ has $\ell$ leaves. Note that $\ell \geq n-d+2 \geq 4$. From Lemma 2.1, $\operatorname{sdiam}_{3}(G) \leq n-\ell+2 \leq n-(n-d+2)+2=d$. Therefore, this graph shows that $e_{3}(n, \ell, d) \leq n-1$. On the other hand, we only consider connected graphs, which implies $e_{3}(n, \ell, d) \geq n-1$. So $e_{3}(n, \ell, d)=n-1$.

The following result is from [37].

Lemma 2.3 [37] Let $G$ be a connected graph of order $n(n \geq 3)$. Then $\operatorname{sdiam}_{3}(G)=n-1$ if and only if $G$ satisfies one of the following conditions.

(i) $G=T_{a, b, c}$, where $T_{a, b, c}$ is a tree with a vertex $v$ of degree 3 such that $T_{a, b, c}-v=$ $P_{a} \cup P_{b} \cup P_{c}$, where $0 \leq a \leq b \leq c$.

(ii) $G=C_{3}(a, b, c)$, where $C_{3}(a, b, c)$ is a graph containing a triangle $K_{3}$ and satisfying $C_{3}(a, b, c)-V\left(K_{3}\right)=P_{a} \cup P_{b} \cup P_{c}$, where $0 \leq a \leq b \leq c$.

Corollary 2.1 Let $G$ be a connected graph of order $n$. If $\operatorname{sdiam}_{3}(G) \leq n-2$ and $\Delta(G)=$ 2 , then $G$ is cycle of order $n$.

Lemma 2.4 37] Let $G$ be a connected graph of order $n$. Then sdiam ${ }_{3}(G)=2$ if and only if $0 \leq \Delta(\bar{G}) \leq 1$ if and only if $n-2 \leq \delta(G) \leq n-1$.

\section{$3 \quad$ For small $d$}

From Theorem 1.1, we have $2 \leq d \leq n-1$. In this section, we discuss the cases $d=2$ and $d=3$. 


\subsection{The case $d=2$}

If $\operatorname{sidam}_{3}(G)=2$, then it follows from Lemma 2.4 that

$$
n-2 \leq \delta(G) \leq n-1
$$

and hence $n-2 \leq \Delta(G) \leq n-1$. So we assume that $n-2 \leq \ell \leq n-1$.

Theorem 3.1 (1) For $\ell=n-1, e_{3}(n, \ell, 2)=\left(\begin{array}{l}n \\ 2\end{array}\right)-\frac{n-1}{2}$ for $n$ odd; $e_{3}(n, \ell, 2)=\left(\begin{array}{l}n \\ 2\end{array}\right)-\frac{n-2}{2}$ for $n$ even.

(2) For $\ell=n-2, e_{3}(n, \ell, 2)=\left(\begin{array}{l}n \\ 2\end{array}\right)-\frac{n}{2}$ for $n$ even; $e_{3}(n, \ell, 2)=\infty$ for $n$ odd.

Proof. (1) For $n$ odd, we let $G$ be a graph obtained from a complete graph $K_{n}$ by deleting a maximum matching $M$. Clearly, $|M|=\frac{n-1}{2}$ and $\Delta(G)=n-1$. From Lemma 2.4. we have $\operatorname{sdiam}_{3}(G)=2$, and hence $e_{3}(n, n-1,2) \leq\left(\begin{array}{l}n \\ 2\end{array}\right)-\frac{n-1}{2}$ for $n$ odd. We claim that $e_{3}(n, n-1,2)=\left(\begin{array}{l}n \\ 2\end{array}\right)-\frac{n-1}{2}$. Assume, to the contrary, that $e_{3}(n, n-1,2) \leq$ $\left(\begin{array}{l}n \\ 2\end{array}\right)-\frac{n-1}{2}-1$. Then there exists a graph $G$ such that $\operatorname{siam}_{3}(G)=2, \Delta(G)=n-1$ and $e(G) \leq\left(\begin{array}{c}n \\ 2\end{array}\right)-\frac{n-1}{2}-1$. Clearly, $\Delta(\bar{G}) \geq 2$ and hence $\delta(G)=n-1-\Delta(\bar{G}) \leq n-3$, which contradicts to (4.1). So $e_{3}(n, n-1,2)=\left(\begin{array}{l}n \\ 2\end{array}\right)-\frac{n-1}{2}$.

For $n$ even, let $G$ be a graph obtained from a complete graph $K_{n}$ by deleting a matching $M$ such that $|M|=\frac{n-2}{2}$. Obviously, $\Delta(G)=n-1$. From Lemma 2.4, we have $\operatorname{sdiam}_{3}(G)=2$, and hence $e_{3}(n, n-2,2) \leq\left(\begin{array}{l}n \\ 2\end{array}\right)-\frac{n-2}{2}$ for $n$ even. We claim that $e_{3}(n, n-2,2)=\left(\begin{array}{l}n \\ 2\end{array}\right)-\frac{n-2}{2}$. Assume, to the contrary, that $e_{3}(n, n-1,2) \leq\left(\begin{array}{c}n \\ 2\end{array}\right)-\frac{n-2}{2}-1$. Then there exists a graph $G$ such that $\operatorname{sdiam}_{3}(G)=2, \Delta(G)=n-1$ and $e(G) \leq$ $\left(\begin{array}{l}n \\ 2\end{array}\right)-\frac{n-2}{2}-1$. Clearly, $\Delta(\bar{G}) \geq 1, \delta(G)=n-1-\Delta(\bar{G}) \leq n-2$, and hence $\delta(G)=n-2$ by (4.1). Since $e(G) \leq\left(\begin{array}{l}n \\ 2\end{array}\right)-\frac{n}{2}$ and $n$ is even, it follows that $G$ is a graph obtained from a complete graph $K_{n}$ by deleting a perfect matching, which implies $\Delta(G)=n-2$, a contradiction. So $e_{3}(n, n-1,2)=\left(\begin{array}{l}n \\ 2\end{array}\right)-\frac{n-1}{2}$.

(2) For $n$ even, let $G$ be a graph obtained from a complete graph $K_{n}$ by deleting a perfect matching $M$. Obviously, $|M|=\frac{n}{2}, \Delta(G)=n-2$ and $\operatorname{sdiam}_{3}(G)=2$. Therefore, $e_{3}(n, n-2,2) \leq\left(\begin{array}{l}n \\ 2\end{array}\right)-\frac{n}{2}$ for $n$ even. We claim that $e_{3}(n, n-2,2)=\left(\begin{array}{l}n \\ 2\end{array}\right)-\frac{n}{2}$. Assume, to the contrary, that $e_{3}(n, n-2,2) \leq\left(\begin{array}{c}n \\ 2\end{array}\right)-\frac{n}{2}-1$. Then there exists a graph $G$ such that $\operatorname{sdiam}_{3}(G)=2, \Delta(G)=n-2$ and $e(G) \leq\left(\begin{array}{l}n \\ 2\end{array}\right)-\frac{n}{2}-1$. Clearly, $\Delta(\bar{G}) \geq 2$, $\delta(G)=n-1-\Delta(\bar{G}) \leq n-3$, a contradiction. So $e_{3}(n, n-1,2)=\left(\begin{array}{l}n \\ 2\end{array}\right)-\frac{n}{2}$.

For $n$ odd, let $G$ be a graph such that $\operatorname{sdiam}_{3}(G)=2$ and $\Delta(G)=n-2$. Since $\operatorname{sdiam}_{3}(G)=2$, it follows that $n-2 \leq \delta(G) \leq n-1$. Since $\Delta(G)=n-2$, it follows that $\delta(G)=\Delta(G)=n-2$, and hence $G$ is $(n-2)$-regular graph of order $n$, which is impossible. So $e_{3}(n, n-2,2)=\infty$ for $n$ odd. 


\subsection{The case $d=3$}

The following proposition and lemma are immediate.

Proposition 3.1 Let $K_{n_{1}, n_{2}, \cdots, n_{r}}$ be a complete $r$-partite graph with $n_{1} \leq n_{2} \leq \cdots \leq n_{r}$. Then

$$
\operatorname{sdiam}_{k}\left(K_{n_{1}, n_{2}, \cdots, n_{r}}\right)= \begin{cases}k-1, & \text { if } k>n_{r} \\ k, & \text { if } k \leq n_{r} .\end{cases}
$$

Proof. Set $G=K_{n_{1}, n_{2}, \cdots, n_{r}}$. Let $V_{1}, V_{2}, \cdots, V_{r}$ be the parts of complete $r$-partite graph $G$, and set $\left|V_{i}\right|=n_{i}(1 \leq i \leq r)$. Suppose $k>n_{r}$. Since $n_{1} \leq n_{2} \leq \cdots \leq n_{r}$, it follows that for any $S \subseteq V(G)$ and $|S|=k$, there exist two parts $V_{i}, V_{j}$ such that $S \cap V_{i} \neq \varnothing$ and $S \cap V_{j} \neq \varnothing$. Set $S=\left\{v_{1}, v_{2}, \cdots, v_{k}\right\}$. Without loss of generality, let $S \cap V_{i}=\left\{v_{1}, v_{2}, \cdots, v_{s}\right\}$ and $S \cap V_{j}=\left\{v_{s+1}, v_{s+2}, \cdots, v_{t}\right\}$. Then the tree induced by the edges in

$$
\left\{v_{1} v_{i} \mid s+1 \leq i \leq k\right\} \cup\left\{v_{s+1} v_{i} \mid 2 \leq i \leq s\right\}
$$

is an $S$-Steiner tree, and hence $d_{G}(S) \leq k-1$, and hence $\operatorname{sdiam}_{k}(G) \leq k-1$. From Theorem 1.1, we have $\operatorname{sdiam}_{k}(G)=k-1$.

Suppose $k \leq n_{r}$. Choose $S \subseteq V(G)$ and $|S|=k$ such that $S \subseteq V_{r}$. Observe that any $S$-Steiner tree must use at least $k$ edges. Therefore, $d_{G}(S) \geq k$, and hence $\operatorname{sdiam}_{k}(G) \geq k$.

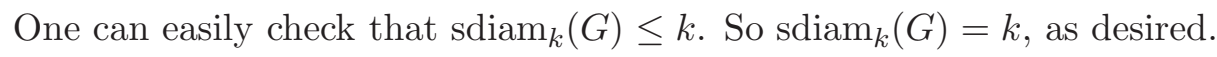

Lemma 3.1 Let $T$ be a tree of order $n(n \geq 5)$. Then sdiam ${ }_{3}(T)=3$ if and only if $T$ is a star.

Proof. If $T$ is a star, then $\operatorname{sdiam}_{3}(T)=3$. Conversely, we $\operatorname{suppose} \operatorname{sdiam}_{3}(T)=3$. Suppose that $T$ contains a path $P$ as its subgraph such that $|V(P)| \geq 3$ and each vertex in $P$ is an internal vertex of $T$. Let $u, v$ be two endpoints of $P$. Then there exit two leaves, say $x, y$, such that $x u \in E(T)$ and $y w \in E(T)$. Choose $S=\{x, u, y\}$. Then $d_{G}(S) \geq 4$ and hence $\operatorname{sdiam}_{3}(T) \geq 4$, a contradiction. Suppose that $T$ has exactly two internal vertices of $T$. Since $n \geq 5$, it follows that $T$ has at least three leaves. Choose three of them as $S$. Then $d_{G}(S) \geq 4$ and hence $\operatorname{sdiam}_{3}(T) \geq 4$, a contradiction. We conclude that $T$ has exactly one internal vertex and hence $T$ is a star.

We first give an upper bound of our parameter for general $\ell$ and $d=3$.

Lemma 3.2 For $\frac{n}{2} \leq \ell \leq n-4$,

$$
n \leq e_{3}(n, \ell, 3) \leq \ell(n-\ell) .
$$

Proof. Let $K_{\ell, n-\ell}$ be a complete bipartite graph. Since $\ell \geq \frac{n}{2}$, it follows that $\ell \geq n-\ell$, and hence $\Delta(G)=\ell$. From Proposition 3.1, we have $\operatorname{sdiam}_{3}(G)=3$. So $e_{3}(n, \ell, 3) \leq \ell(n-\ell)$. 
Let $G$ be a graph with $\operatorname{sdiam}_{3}(G) \leq 3$ and $\frac{n}{2} \leq \Delta(G)=\ell \leq n-4$. If $\operatorname{sdiam}_{3}(G)=2$, then it follows from Lemma 2.4 that $n-3 \leq \delta(G) \leq n-2$, which contradicts $\Delta(G)=\ell \leq n-4$. Suppose $\operatorname{sdiam}_{3}(G)=3$. From Lemma 3.1, if $G$ is tree, then $G$ is a star, and hence $\Delta(G)=n-1$, a contradiction. So $e_{3}(n, \ell, 3) \geq n$.

Lemma 3.3 For $\ell=n-1, e_{3}(n, \ell, 3)=n-1$.

Proof. For $\ell=n-1$, let $G$ be a star of order $n$. Then $\operatorname{sdiam}_{3}(G)=3$ and $\Delta(G)=n-1$, and hence $e_{3}(n, n-1,3) \leq n-1$. Since we only consider the connected graph, it follows that $e_{3}(n, n-1,3) \geq n-1$. So $e_{3}(n, n-1,3)=n-1$.

Lemma 3.4 For $\ell=n-2, e_{3}(n, \ell, 3)=2 n-5$.

Proof. For $\ell=n-2$, let $G=K_{2, n-2}^{-}$be a graph obtained from a complete bipartite graph $K_{2, n-2}$ by deleting an edge; see Figure $3.1(a)$. Let $u, v, x_{n-2}$ be the vertices of degree $n-1, n-2,1$ in $K_{2, n-2}^{-}$, respectively. Set $X=V(G)-\left\{u, v, x_{n-2}\right\}=\left\{x_{1}, x_{2}, \cdots, x_{n-3}\right\}$.

Now, we show $\operatorname{sidam}_{3}(G) \leq 3$. It suffices to prove that $d_{G}(S) \leq 3$ for any $S \subseteq V(G)$ and $|S|=3$. If $|S \cap X|=3$, without loss of generality, let $S=\left\{x_{1}, x_{2}, x_{3}\right\}$, then the tree $T$ induced by the edges in $\left\{u x_{1}, u x_{2}, u x_{3}\right\}$ is an $S$-Steiner tree and hence $d_{G}(S) \leq$ 3. If $|S \cap X|=0$, then $S=\left\{u, v, x_{n-2}\right\}$, then the tree $T$ induced by the edges in $\left\{u x_{1}, v x_{1}, u x_{n-2}\right\}$ is an $S$-Steiner tree and hence $d_{G}(S) \leq 3$. Suppose $|S \cap X|=2$. Then $\left|S \cap\left\{u, v, x_{n-2}\right\}\right|=1$. Without loss of generality, let $S=\left\{x_{1}, x_{2}, u\right\}$ or $S=\left\{x_{1}, x_{2}, v\right\}$ or $S=\left\{x_{1}, x_{2}, x_{n-2}\right\}$. If $S=\left\{x_{1}, x_{2}, u\right\}$, then the tree $T_{1}$ induced by the edges in $\left\{u x_{1}, u x_{2}\right\}$ is a Steiner tree connecting $\left\{x_{1}, x_{2}, u\right\}$. If $S=\left\{x_{1}, x_{2}, v\right\}$, then the tree $T_{2}$ induced by the edges in $\left\{v x_{1}, v x_{2}\right\}$ is a Steiner tree connecting $\left\{x_{1}, x_{2}, v\right\}$. If $\left\{x_{1}, x_{2}, x_{n-2}\right\}$, then the tree $T_{3}$ induced by the edges in $\left\{u x_{1}, u x_{2}, u x_{n-2}\right\}$ is a Steiner tree connecting $\left\{x_{1}, x_{2}, x_{n-2}\right\}$. Therefore, $d_{G}(S) \leq 3$. Suppose $|S \cap X|=1$. Then $\left|S \cap\left\{u, v, x_{n-2}\right\}\right|=2$. Without loss of generality, let $S=\left\{x_{1}, u, v\right\}$ or $S=\left\{x_{1}, u, x_{n-2}\right\}$ or $S=\left\{x_{1}, v, x_{n-2}\right\}$. If $S=\left\{x_{1}, u, v\right\}$, then the tree $T_{1}$ induced by the edges in $\left\{u x_{1}, v x_{1}\right\}$ is a Steiner tree connecting $\left\{x_{1}, u, v\right\}$. If $\left\{x_{1}, u, x_{n-2}\right\}$, then the tree $T_{2}$ induced by the edges in $\left\{v x_{1}, u x_{1}, u x_{n-2}\right\}$ is a Steiner tree connecting $\left\{x_{1}, u, x_{n-2}\right\}$. If $\left\{x_{1}, v, x_{n-2}\right\}$, then the tree $T_{2}$ induced by the edges in $\left\{u x_{1}, u x_{n-2}\right\}$ is a Steiner tree connecting $\left\{x_{1}, v, x_{n-2}\right\}$. Therefore, $d_{G}(S) \leq 3$. From the above argument, we conclude that $d_{G}(S) \leq 3$ for any $S \subseteq V(G)$ and $|S|=3$. Since $\operatorname{sidiam}_{3}(G) \leq 3$ and $\Delta(G)=n-2$, we have $e_{3}(n, n-2,3) \leq 2 n-5$.

We now show $e_{3}(n, n-2,3) \geq 2 n-5$. Let $G$ be a graph with $\Delta(G)=n-2$ and $\operatorname{sdiam}(G) \leq 3$. Since $\Delta(G)=\ell=n-2$, there exists a vertex $u$ such that $d_{G}(u)=n-2$. Then $\left|N_{G}(u)\right|=n-2$ and $\{u\} \cup N_{G}(u) \subseteq V(G)$. Clearly, there is a vertex $v \in V(G)$ such that $u v \notin E(G)$. Let $X^{\prime}=V(G) \backslash\{u, v\}=\left\{x_{1}, x_{2}, \cdots, x_{n-2}\right\}$. Clearly, $\left|X^{\prime}\right| \geq 3$. Since $G$ is connected, it follows that there exists a vertex, say $x_{j} \in X$ such that $v x_{j} \in E(G)$. 


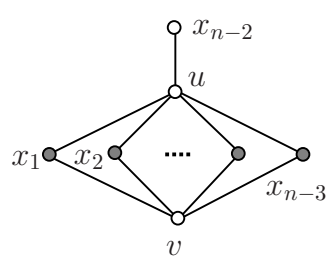

(a)

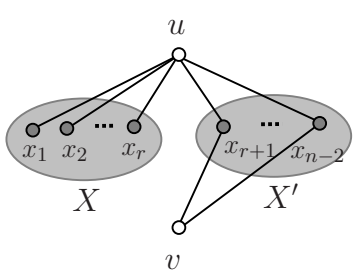

(b)

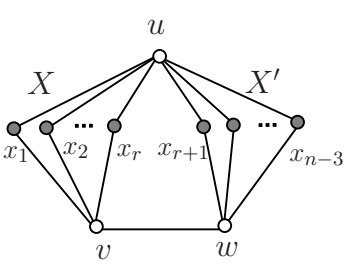

(c)

Figure 3.1 Graphs for Lemma 3.4.

Without loss of generality, let $v x_{n-2} \in E(G)$. Observe that for each $x_{i}(1 \leq i \leq n-3)$ we have $v x_{i} \in E(G)$ or $v x_{i} \notin E(G)$. Without loss of generality, let $v x_{1}, v x_{2}, \cdots, v x_{r} \notin E(G)$ and $v x_{r+1}, v x_{r+2}, \cdots, v x_{n-3} \in E(G)$, where $0 \leq r \leq n-3$. Set $X=\left\{x_{1}, x_{2}, \cdots, x_{r}\right\}$. For each two vertices $x_{i}, x_{j} \in X$, we choose $S=\left\{x_{i}, x_{j}, v\right\}$. Since $\operatorname{sdiam}_{3}(G) \leq 3$, it follows that any $S$-Steiner tree contains at most 3 edges. Therefore, there exists a vertex $x_{k} \in\left\{x_{r+1}, x_{r+2}, \cdots, x_{n-3}\right\}$ such that $x_{i} x_{k}, x_{j} x_{k} \in E(G)$ or $x_{i} x_{j}, x_{j} x_{k} \in E(G)$ or $x_{i} x_{j}, x_{i} x_{k} \in E(G)$. For $r$ even, $e(G) \geq(n-2)+(n-2-r)+r=2 n-4$. For $r$ odd, $e(G) \geq(n-2)+(n-2-r)+(r-1)=2 n-5$. So $e_{3}(n, n-2,3) \geq 2 n-5$.

From the above argument, we conclude that $e_{3}(n, n-2,3)=2 n-5$.

Lemma 3.5 For $\ell=n-3, e_{3}(n, n-3,3)=2 n-5$.

Proof. Let $G$ be a graph defined as follows; see Figure $3.1(c)$.

$$
\begin{aligned}
V(G)= & \{u, v, w\} \cup\left\{x_{i} \mid 1 \leq i \leq n-3\right\} \\
E(G)= & \left\{u x_{i} \mid 1 \leq i \leq n-3\right\} \cup\left\{v x_{i} \mid 1 \leq i \leq r\right\} \\
& \cup\left\{w x_{i} \mid r+1 \leq i \leq n-3\right\} \cup\{v w\} .
\end{aligned}
$$

One can check that $\operatorname{sdiam}_{3}(G)=3$ and $\Delta(G)=n-3$. Therefore, $e_{3}(n, n-3,3) \leq 2 n-5$.

We only need to show $e_{3}(n, n-3,3) \geq 2 n-5$. Let $G$ be a connected graph with $\Delta(G)=n-3$ and $\operatorname{sdiam}_{3}(G)=3$. It suffices to show that $e(G) \geq 2 n-5$. Since $\Delta(G)=$ $\ell=n-3$, there exists a vertex $u$ such that $d_{G}(u)=n-3$, and hence $\left|N_{G}(u)\right|=n-3$ and $\{u\} \cup N_{G}(u) \subseteq V(G)$. Clearly, there exist two vertices $v, w \in V(G)$ such that $v, w \notin N_{G}(u)$. Let $V(G) \backslash\{u, v, w\}=\left\{x_{1}, x_{2}, \cdots, x_{n-3}\right\}$. We have the following two cases to consider.

Case 1. $v w \in E(G)$

Since $G$ is connected, it follows that there exist two vertices $x_{j}, x_{k}$ such that $v x_{j} \in E(G)$ and $v x_{k} \in E(G)$ (note that $x_{j}, x_{k}$ are not necessarily different). Without loss of generality, let $v x_{1}, w x_{1} \in E(G)$ or $v x_{1}, w x_{2} \in E(G)$. Suppose $v x_{1}, w x_{1} \in E(G)$. For any $x_{i}(2 \leq i \leq$ $n-3)$, we choose $S=\left\{x_{i}, v, w\right\}$. Since $\operatorname{sdiam}_{3}(G)=3$, it follows that $x_{1} x_{i} \in E(G)$ or 
$v x_{i} \in E(G)$ or $w x_{i} \in E(G)$, and hence $e(G) \geq(n-3)+2+(n-4)=2 n-5$, as desired. Suppose $v x_{1}, w x_{2} \in E(G)$. For $x_{3}$, we choose $S=\left\{x_{3}, v, w\right\}$. Since $\operatorname{sdiam}_{3}(G)=3$, it follows that $v x_{3}, w x_{3} \in E(G)$ or $x_{3} x_{1}, w x_{1} \in E(G)$ or $x_{3} x_{2}, v x_{2} \in E(G)$. For any $x_{i}(2 \leq i \leq n-3)$, we choose $S=\left\{x_{i}, v, w\right\}$. Since $\operatorname{sdiam}_{3}(G)=3$, it follows that $x_{1} x_{i} \in E(G)$ or $x_{2} x_{i} \in E(G)$ or $x_{3} x_{i} \in E(G)$ or $v x_{i} \in E(G)$ or $w x_{i} \in E(G)$, and hence $e(G) \geq(n-3)+4+(n-6)=2 n-5$, as desired.

Case 2. $v w \notin E(G)$

Since $G$ is connected, it follows that there exists a vertex $x_{j}$ such that $v x_{j} \in E(G)$ or $w x_{j} \in E(G)$. Without loss of generality, let $v x_{1} \in E(G)$. For any $x_{i}(2 \leq i \leq n-3)$, we choose $S=\left\{x_{i}, v, w\right\}$. Since $\operatorname{sdiam}_{3}(G)=3$, it follows that $x_{1} x_{i} \in E(G)$ or $v x_{i} \in E(G)$ or $w x_{i} \in E(G)$, and hence $e(G) \geq(n-3)+1+1+(n-4)=2 n-5$, as desired.

From the argument, we conclude that $e(G) \geq 2 n-5$, and hence $e_{3}(n, n-3,3) \geq 2 n-5$.

We now conclude our results for $d=3$.

Theorem 3.2 (1) For $\ell=n-1, e_{3}(n, n-1,3)=n-1$;

(2) For $\ell=n-2, e_{3}(n, n-2,3)=2 n-5$;

(3) For $\ell=n-3, e_{3}(n, n-3,3)=2 n-5$;

(4) For $\ell=2, e_{3}(n, 2,3)=3$ for $n=4 ; e_{3}(n, 2,3)=5$ for $n=5 ; e_{3}(n, 2,3)=\infty$ for $n \geq 6$.

(5) For $\frac{n}{2} \leq \ell \leq n-4, n \leq e_{3}(n, \ell, 3) \leq \ell(n-\ell)$.

Proof. The results in (1)-(3) follow from Lemmas 3.3, 3.4 and 3.5, Let $G$ be a connected graph with $\Delta(G)=2$ and $\operatorname{sdiam}_{3}(G)=3$. Then $G=P_{n}$ or $G=C_{n}$. If $G=P_{n}$, then it follows from Lemma 2.3 that $3=\operatorname{sdiam}_{3}(G)=\operatorname{sdiam}_{3}\left(P_{n}\right)=n-1$, and hence $n=4$. If $G=C_{n}$, then it follows from Observation 2.1 that $3=\operatorname{sdiam}_{3}(G)=\operatorname{sdiam}_{3}\left(C_{n}\right)=\left\lfloor\frac{2 n}{3}\right\rfloor$, and hence $n=5$. Furthermore, $e_{3}(n, 2,3)=\infty$ for $n \geq 6$. The result in (5) follow from Lemma 3.2 ,

\section{For large $d$}

For $d=n-1$ and $4 \leq \ell \leq n-1$, we have proved that $e_{3}(n, \ell, n-1)=n-1$. We study the cases $k=n-2, n-3, n-4$ in this section.

Let $T(a, b, c)$ be a tree obtained from two stars $K_{1, a}, K_{1, c}$ and a path $P=v_{1} v_{2} \ldots v_{b}$ by identifying $u$ and $v_{1}, w$ and $v_{b}$, where $a+b+c=n, u$ is the center of $K_{1, a}$, and $w$ is the center of $K_{1, c}$. Let $T^{*}(x, y)$ be a tree obtained from three stars $K_{1,3}, K_{1,3}, K_{1, y}$ and a path $P=v_{1} v_{2} \ldots v_{b}$ by identifying $u, v, v_{1}$ and $w, v_{b}$, where $x+y+6=n, u$ is a leaf of $K_{1,3}$, and $v$ is a leaf of another $K_{1,3}$, and $w$ is the center of $K_{1, c}$. 


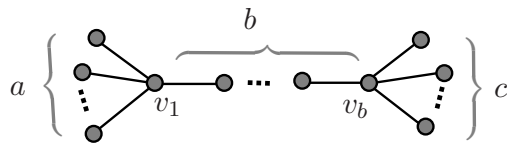

(a) $T(a, b, c)$

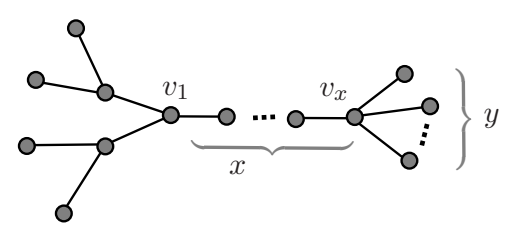

(b) $T^{*}(x, y)$

Figure 4.1 Graphs for $n \geq 8$ in Lemma 4.2.

\subsection{The case $d=n-2$}

In this subsection, we study the case $d=n-2$.

Theorem 4.1 (1) For $n \geq 4, e_{3}(n, 2, n-2)=n$.

(2) For $n \geq 4$,

$$
e_{3}(n, 3, n-2)= \begin{cases}n+1 & \text { if } n=4 \\ n & \text { if } n=5 \\ n-1 & \text { if } n \geq 6\end{cases}
$$

(3) For $n \geq 5$ and $4 \leq \ell \leq n-1, e_{3}(n, \ell, n-2)=n-1$.

Proof. (1) For $n \geq 4$, let $G=C_{n}$ be a cycle of order $n$. Then $\ell=\Delta(G)=2$. From Observation 2.1, we have $\operatorname{sidiam}_{3}(G) \leq n-2$ and hence this graph shows $e_{3}(n, 2, n-2) \leq n$. It suffices to show that $e_{3}(n, 2, n-2) \geq n$. Let $G$ be a graph with $\Delta(G)=2$ and $\operatorname{sdiam}_{3}(G) \leq n-2$. Since $\Delta(G)=\ell=2$, it follows that $G=P_{n}$ or $G=C_{n}$. From Lemma 2.3. we have $\operatorname{sdiam}_{3}\left(P_{n}\right)=n-1$ and $\operatorname{sdiam}_{3}\left(C_{n}\right) \leq n-2$. So $e_{3}(n, 2, n-2) \geq n$ and hence $e_{3}(n, 2, n-2)=n$.

(2) For $n \geq 6$, let $G$ be a graph obtained from three paths $P_{1}=u_{1} u_{2} u_{3}, P_{2}=w_{1} w_{2} w_{3}$ and $P_{3}=v_{1} v_{2} \cdots v_{n-4}$ by identifying the vertices $u_{2}$ and $v_{1}$, and then identifying the vertices $w_{2}$ and $v_{n-4}$. Clearly, $\ell=\Delta(G)=3$. From Lemma 2.3 , we have $\operatorname{sdiam}_{3}(G) \leq$ $n-2$. Therefore, $e_{3}(n, 3, n-2) \leq n-1$ and hence $e_{3}(n, 3, n-2)=n-1$. For $n=4$, the graph $G=K_{4}^{-}$shows that $e_{3}(4,3,2) \leq 5$. It suffices to show that $e_{3}(4,3,2) \geq 5$. Let $G$ be a graph with $\Delta(G)=2$ and $\operatorname{sdiam}_{3}(G) \leq n-2=2$. Since $\ell=\Delta(G)=3$, there exists a vertex of degree 3 , say $u_{1}$. Let $u_{1} u_{2}, u_{1} u_{3}, u_{1} u_{4} \in E(G)$. Choose $S=\left\{u_{2}, u_{3}, u_{4}\right\}$. Since $\operatorname{sdiam}_{3}(G)=2$, it follows that $u_{2} u_{3}, u_{3} u_{4} \in E(G)$. Therefore, $e(G) \geq 5$ and hence $e_{3}(4,3,2) \geq 5$. So $e_{3}(4,3,2)=5$. For $n=5$, let $G$ be a graph obtained from a cycle $C_{4}$ by adding a pendent edge at one vertex of $C_{4}$. One can check that $\Delta(G)=3$ and $\operatorname{sdiam}_{3}(G) \leq 3$. Therefore, $e_{3}(5,3,3) \leq 5$. We need to show $e_{3}(5,3,3) \geq 5$. Let $G$ be a graph with $\Delta(G)=3$ and $\operatorname{sdiam}_{3}(G) \leq n-2=3$. Since $\ell=\Delta(G)=3$, there exists a vertex of degree 3 , say $u_{1}$. Let $u_{1} u_{2}, u_{1} u_{3}, u_{1} u_{4} \in E(G)$. Since $n=5$, it follows that there 
exists a vertex $u_{5} \in V(G)$. Furthermore, there exists some vertex $u_{j}(2 \leq j \leq 4)$ such that $u_{j} u_{5} \in E(G)$. Without loss of generality, let $u_{2} u_{5} \in E(G)$. Choose $S=\left\{u_{3}, u_{4}, u_{5}\right\}$. Since $\operatorname{sdiam}_{3}(G) \leq 3$, it follows that $u_{3} u_{5} \in E(G)$, or $u_{4} u_{5} \in E(G)$, or $u_{2} u_{3}, u_{2} u_{4} \in E(G)$. Therefore, $e(G) \geq 5$ and hence $e_{3}(5,3,3) \geq 5$, as desired. So $e_{3}(5,3,3)=5$.

(3) For $n \geq 5$ and $4 \leq \ell \leq n-1$, let $G$ be a graph obtained from a star $S_{\ell}$ and a path $P_{n-\ell+1}$ by identifying the center of the star and one end of the path. Clearly, $\Delta(G)=\ell$. From Lemma 2.3, we have $\operatorname{sdiam}_{3}(G) \leq n-2$ and hence this graph shows $e_{3}(n, \ell, n-2) \leq$ $n-1$. Since we only consider connected graphs, we have $e_{3}(n, \ell, n-2) \geq n-1$. So $e_{3}(n, \ell, n-2)=n-1$.

\subsection{The case $d=n-3$}

Let us now turn to the case $d=n-3$.

Lemma 4.1 For $n \geq 5$,

$$
e_{3}(n, 2, n-3)= \begin{cases}\infty & \text { if } n=5,6, \\ n & \text { if } n \geq 7 .\end{cases}
$$

Proof. For $n \geq 7$ and $\ell=2$, let $G=C_{n}$ be a cycle of order $n$. From Observation 2.1, we have $\operatorname{sdiam}_{3}(G)=\left\lfloor\frac{2 n}{3}\right\rfloor \leq n-3$ and hence this graph shows $e_{3}(n, 2, n-3) \leq n$. It suffices to prove $e_{3}(n, 2, n-3) \geq n$. Let $G$ be a connected graph with $\Delta(G)=2$ and $\operatorname{sdiam}_{3}(G) \leq n-3$. From Corollary 2.1, we have $G=C_{n}$ and hence $e_{3}(n, 2, n-3) \geq n$. So $e_{3}(n, 2, n-3)=n$. For $n=5,6$, we have $G=C_{n}$ by Corollary 2.1. From Observation 2.1. we have $\operatorname{sdiam}_{3}(G)=\left\lfloor\frac{2 n}{3}\right\rfloor>n-3$. So $e_{3}(n, 2, n-3)=\infty$ for $n=5,6$.

Lemma 4.2 For $n \geq 5$,

$$
e_{3}(n, 3, n-3)= \begin{cases}\infty & \text { if } n=5 \\ n+1 & \text { if } n=6,7 \\ n-1 & \text { if } n \geq 8\end{cases}
$$

Proof. For $n \geq 8$, let $G=T^{*}(n-7,1)$. Then $G$ has exactly 5 leaves and $\Delta\left(T^{\prime}\right)=3$. From Lemma 2.1, $\operatorname{sdiam}_{3}\left(T^{\prime}\right) \leq n-3$. This tree shows that $e_{3}(n, 3, n-3) \leq n-1$ and hence $e_{3}(n, 3, n-3)=n-1$. For $n=5$, let $G$ be a graph with $\Delta(G)=3$ and $\operatorname{sdiam}_{3}(G) \leq n-3=2$. Furthermore, $\operatorname{sdiam}_{3}(G)=2$. From Lemma 2.4, we have $3 \leq \delta(G) \leq 4$ and hence $3 \leq \delta(G) \leq \Delta(G)=3$, which implies that $G$ is 3-regular. The degree sum of graph $G$ is exactly 15 , a contradiction. So $e_{3}(5,3,2)=\infty$.

For $n=6$, we let $G=A_{4}$; see Figure $4.2(d)$. One can check that $\Delta(G)=3$ and $\operatorname{sdiam}_{3}(G) \leq n-3=3$. Then $e_{3}(6,3,3) \leq 7$. It suffices to show $e_{3}(6,3,3) \geq 7$. Let $G$ be 
a graph such that $\Delta(G)=3$ and $\operatorname{sdiam}_{3}(G) \leq 3$. If $G$ is a tree, then $G=A_{1}$ or $G=A_{2}$ or $G=A_{3}$; see Figure $4.2(a),(b),(c)$. Clearly, if we choose vertex set $S$ consisting of three black vertices, then $d_{G}(S)>3$, which results in $\operatorname{sdiam}_{3}(G)>3$, a contradiction. So $G$ contains at least one cycle. Furthermore, we have the following claim.

Claim 1. $G$ contains at least two cycles.

Proof of Claim 1. Assume, to the contrary, that $G$ is a unicyclic graph. Let $c(G)$ be the circumference of graph $G$. Clearly, $3 \leq c(G) \leq 6$. If $c(G)=6$, then $G=C_{6}$, which contradicts to $\Delta(G)=3$. We may assume that $3 \leq c(G) \leq 5$. If $c(G)=5$, then $G=A_{5}$; see Figure $4.2(e)$. One can also check that $\operatorname{sdiam}_{3}(G)>3$, also a contradiction. If $c(G)=4$, then $G=A_{6}$ or $G=A_{7}$ or $G=A_{8}$; see Figure $4.2(f),(g),(h)$. One can check that $\operatorname{sdiam}_{3}(G)>3$, a contradiction. For $c(G)=3$, one can also check that $\operatorname{sdiam}_{3}(G)>3$, also a contradiction.

From Claim 1, $G$ contains at least two cycles and hence $e(G) \geq 7$. So $e_{3}(6,3,3) \geq 7$, as desired.

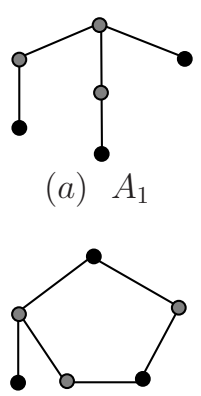

(e) $A_{5}$

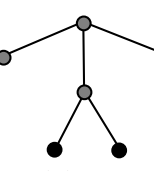

(b) $A_{2}$

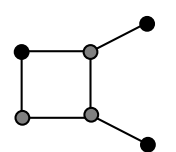

(f) $A_{6}$

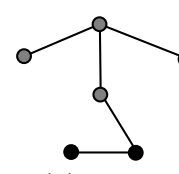

(c) $A_{3}$

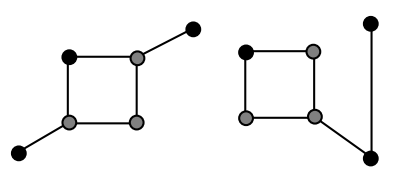

(g) $A_{7}$

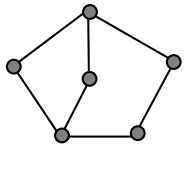

(d) $A_{4}$

(h) $A_{8}$

Figure 4.2 Graphs for $n \geq 8$ in Lemma 4.2 .

For $n=7$, we let $G=B_{1}$; see Figure $4.3(a)$. Then $\Delta(G)=3$ and $\operatorname{sdiam}_{3}(G) \leq$ $n-3=4$. This graph shows that $e_{3}(7,3,4) \leq 8$. It suffices to show that $e_{3}(7,3,4) \geq 8$. Let $G$ be connected graph of order $n$ with $\operatorname{sdiam}_{3}(G) \leq 4$ and $\Delta(G)=3$.

Claim 2. $G$ contains at least two cycles.

Proof of Claim 2. Assume, to the contrary, that $G$ is a tree or has exactly one cycle. If $G$ is a tree, then $G \in\left\{B_{i} \mid 10 \leq i \leq 14\right\}$, and hence $\operatorname{sdiam}_{3}(G)>5$ by choosing the three black vertices as $S$, a contradiction. We now suppose that $G$ contains exactly one cycle. Let $c(G)$ denote the circumference of $G$. Clearly, $3 \leq c(G) \leq 7$. If $c(G)=7$, then $G=C_{7}$, which contradicts to $\Delta(G)=3$. If $c(G)=6$, then $G=B_{2}$; see Figure 4.3 (b). By choosing the three black vertices as $S$, we can see that $\operatorname{sdiam}_{3}(G)>5$, a contradiction. If $c(G)=5$, then $G=B_{3}$ or $G=B_{4}$ or $G=B_{5}$; see Figure $4.3(b)$. By choosing the 
three black vertices as $S$, we can see that $\operatorname{sdiam}_{3}(G)>5$, a contradiction. If $c(G)=4$, then $G \in\left\{B_{i} \mid 6 \leq i \leq 9\right\}$; see Figure 4.3 (b). By choosing the three black vertices as $S$, we can see that $\operatorname{sdiam}_{3}(G)>5$, a contradiction. For $c(G)=3$, one can also check that $\operatorname{sdiam}_{3}(G)>5$, also a contradiction.

From Claim 2, $G$ contains at least two cycles, and hence $e(G) \geq 8$. So $e_{3}(n, 3,4)=$ $e(G) \geq 8$, as desired.

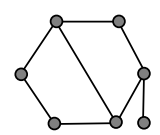

(a) $B_{1}$
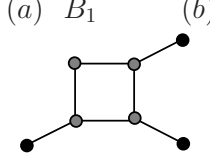

(f) $B_{6}$

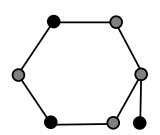

(b) $B_{2}$

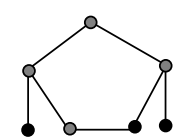

(c) $B_{3}$

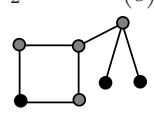

(g) $B_{7}$

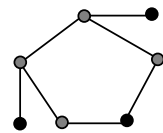

(d) $B_{4}$

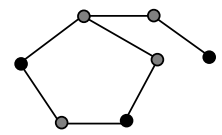

(e) $B_{5}$

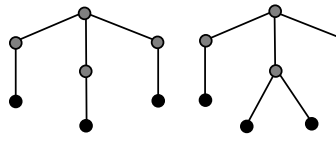

(j) $B_{10}$

(k) $B_{11}$

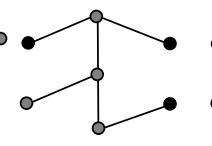

(l) $B_{12}$

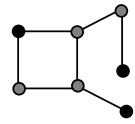

(h) $B_{8}$

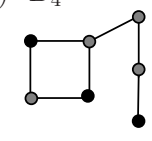

(i) $B_{9}$

Figure 4.3 Graphs for $n=7$ in Lemma 3.2

Lemma 4.3 For $n \geq 5$,

$$
e_{3}(n, 4, n-3)= \begin{cases}\left(\begin{array}{l}
n \\
2
\end{array}\right)-2 & \text { if } n=5 \\
n+1 & \text { if } n=6 \\
n-1 & \text { if } n \geq 7\end{cases}
$$

Proof. For $n \geq 7$, let $G=T(3,2,2)$. The the tree $T(3,2,2)$ have exactly 5 leaves. Clearly, $\Delta(T(3,2,2))=4$. From Lemma 2.1, $\operatorname{siam}_{3}(T(3,2,2)) \leq n-3$. This tree shows that $e_{3}(n, 4, n-3) \leq n-1$, and hence $e_{3}(n, 4, n-3)=n-1$. For $n=5$, let $G$ be the graph obtained from a complete graph $K_{5}$ by deleting a maximum matching. It is clear that $\Delta(G)=4$ and $\operatorname{sdiam}_{3}(G)=2=n-3$. This graph shows that $e_{3}(5,4,2) \leq\left(\begin{array}{l}5 \\ 2\end{array}\right)-2=8$. We need to show that $e_{3}(5,4,2) \geq 8$. Let $G$ be a graph with $\Delta(G)=4$ and $\operatorname{sdiam}_{3}(G) \leq$ $n-3=2$. From Lemma 2.4, we have $3 \leq \delta(G) \leq 4$. From this together with $\Delta(G)=4$ and $\operatorname{sdiam}_{3}(G)=2$, it follows that $e(G) \geq 8$ and hence $e_{3}(5,4,2) \geq 8$, as desired. So $e_{3}(5,4,2)=8$.

For $n=6$, the graph $D_{1}$ shown in Figure $4.4(a)$ satisfies $\Delta\left(H_{1}\right)=4$ and $\operatorname{sdiam}_{3}\left(D_{1}\right) \leq$ $3=n-3$. This graph shows that $e_{3}(6,4,3) \leq 7$. It suffices to prove that $e_{3}(6,4,3) \geq 7$. 


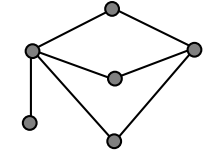

(a) $H_{1}$

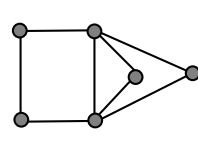

(b) $\mathrm{H}_{3}$

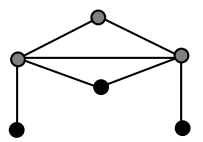

(c) $H_{3}$

Figure 4.4 Graphs for Lemma 4.3 .

Let $G$ be a graph with $\Delta(G)=4$ and $\operatorname{sdiam}_{3}(G) \leq 3$. Since $\Delta(G)=4$, it follows that there exists a vertex of degree 4 , say $u$. Set $N_{G}(u)=\left\{x_{1}, x_{2}, x_{3}, x_{4}\right\}$. Since $n=6$, it follows that there is a remaining vertex in $G$, say $v$. Because $G$ is connected, the vertex $v$ is adjacent to one of $\left\{x_{1}, x_{2}, x_{3}, x_{4}\right\}$. Without loss of generality, let $v x_{4} \in E(G)$. Choose $S=\left\{x_{2}, x_{3}, v\right\}$. Since $\operatorname{sdiam}_{3}(G) \leq 3$, it follows that $x_{2} v \in E(G)$, or $x_{3} v \in E(G)$, or $x_{2} x_{4}, x_{3} x_{4} \in E(G)$. If $x_{2} v \in E(G)$, then we choose $S=\left\{x_{1}, x_{3}, v\right\}$ and hence $x_{1} v \in E(G)$, or $x_{3} v \in E(G)$, or $x_{1} x_{2}, x_{3} x_{2} \in E(G)$, or $x_{1} x_{4}, x_{3} x_{4} \in E(G)$. By symmetry, we only need to consider $x_{1} v \in E(G)$ or $x_{1} x_{2}, x_{3} x_{2} \in E(G)$. For the former, we have $x_{1} v \in E(G)$ and hence $G=D_{1}$; see Figure $4.4(b)$. If $x_{1} x_{2}, x_{3} x_{2} \in E(G)$, then $G=D_{2}$; see Figure $4.4(c)$. The case $x_{3} v \in E(G)$ is just the same as $x_{2} v \in E(G)$, and so we omit its discussion. We may assume that $x_{2} x_{4}, x_{3} x_{4} \in E(G)$. Clearly, $G=D_{3}$; see Figure $4.4(d)$. Choose the three black vertices to form vertex set $S$. Then $d_{G}(S) \geq 4$. So $e_{3}(6,4,3)=7$ if and only if $G \in\left\{D_{1}, D_{2}\right\}$.

From Lemmas 3.1, 3.2, 3.3 and 2.2, we have the following result.

Theorem 4.2 (1) For $n \geq 5$,

$$
e_{3}(n, 2, n-3)= \begin{cases}\infty & \text { if } n=5,6 \\ n & \text { if } n \geq 7 .\end{cases}
$$

(2) For $n \geq 5$,

$$
e_{3}(n, 3, n-3)= \begin{cases}\infty, & \text { if } n=5 \\ n+1 & \text { if } n=6 \\ n & \text { if } n=7 \\ n-1 & \text { if } n \geq 8\end{cases}
$$

(3) For $n \geq 5$,

$$
e_{3}(n, 4, n-3)= \begin{cases}\left(\begin{array}{l}
n \\
2
\end{array}\right)-2 & \text { if } n=5 \\
n+1 & \text { if } n=6 \\
n-1 & \text { if } n \geq 7\end{cases}
$$

(4) For $n \geq 6$ and $5 \leq \ell \leq n-1, e_{3}(n, \ell, n-3)=n-1$. 


\subsection{The case $d=n-4$}

In this subsection, we consider the case $d=n-4$.

Lemma 4.4 For $n \geq 5$,

$$
e_{3}(n, 2, n-4)= \begin{cases}\infty & \text { if } 5 \leq n \leq 9 \\ n & \text { if } n \geq 10\end{cases}
$$

Proof. For $n \geq 10$ and $\ell=2$, let $G=C_{n}$ be a cycle of order $n$. From Observation 2.1. we have $\operatorname{sidam}_{3}(G)=\left\lfloor\frac{2 n}{3}\right\rfloor \leq n-4$ and hence this graph shows $e_{3}(n, 2, n-4) \leq n$. It suffices to prove $e_{3}(n, 2, n-3) \geq n$. Let $G$ be a connected graph with $\Delta(G)=2$ and $\operatorname{sdiam}_{3}(G) \leq n-4$. From Corollary 2.1, we have $G=C_{n}$ and hence $e_{3}(n, 2, n-4) \geq n$. So $e_{3}(n, 2, n-4)=n$. For $5 \leq n \leq 9$, we have $G=C_{n}$ by Corollary 2.1. From Observation 2.1, we have $\operatorname{sdiam}_{3}(G)=\left\lfloor\frac{2 n}{3}\right\rfloor>n-4$. So $e_{3}(n, 2, n-4)=\infty$ for $5 \leq n \leq 9$.

Lemma 4.5 For $n \geq 6$,

$$
e_{3}(n, 3, n-4)= \begin{cases}\infty & \text { if } n=6 \\ n+3 & \text { if } n=7 \\ n+2 & \text { if } n=8 \\ n+1 & \text { if } n=9 \\ n-1 & \text { if } n \geq 10\end{cases}
$$

Proof. For $n \geq 10$, let $T_{3}$ be a tree of maximum degree 3 with exactly 6 leaves. Clearly, $\Delta\left(T^{\prime \prime}\right)=3$. From Lemma 2.1, we have $\operatorname{sdiam}_{3}\left(T_{3}\right) \leq n-4$. This tree shows that $e_{3}(n, 3, n-4) \leq n-1$ and hence $e_{3}(n, 3, n-4)=n-1$. For $n=6$, let $G$ be a graph with $\Delta(G)=3$ and $\operatorname{sdiam}_{3}(G) \leq n-4=2$. From Lemma 2.4, we have $4 \leq \delta(G) \leq 5$, which contradicts to the fact $\Delta(G)=3$. So $e_{3}(6,3,2)=\infty$.

Suppose $7 \leq n \leq 9$. For $n=9$, let $G$ be a graph obtained from a cycle $C=v_{1} v_{2} \ldots v_{9}$ by adding a new edge $v_{1} v_{5}$. One can easily check that $\operatorname{sdiam}_{3}(G) \leq 5=n-4$ and hence this graph shows $e_{3}(9,3,5) \leq 10=n+1$. It suffices to show that $e_{3}(9,3,5) \geq 10=n+1$. Let $G$ be a graph with $\Delta(G)=3$ and $\operatorname{sdiam}_{3}(G) \leq 5$. Suppose $G$ is a tree. We claim that $G$ has at most 5 leaves. Assume, to the contrary, that $G$ has $t(6 \leq t \leq 8)$ leaves. Since $\Delta(G)=3$, it follows that $16=2 e(G)=\sum_{v \in V(G)} d(v) \leq t+3(9-t)=27-2 t \leq 15$, a contradiction. Since $\Delta(G)=3$, it follows that $G$ has at least 3 leaves and at most 5 leaves. If $G$ is a tree with $t(3 \leq t \leq 5)$ leaves, then we choose three of them as $S$, then $d_{G}(S)=11-t \geq 6$, which contradicts to $\operatorname{sdiam}_{3}(G) \leq 5$. We conclude that $G$ is not a tree, and hence $G$ has cycles. Furthermore, we have the following claim.

Claim 1. G contains at least two cycles. 
Proof of Claim 1. Assume, to the contrary, that $G$ has exactly one cycle. Let $x$ be the number of vertices of degree 1 in $G$, and $y$ be the number of vertices of degree 2 in $G$. We claim that $0 \leq x \leq 4$. Assume, to the contrary, that $x \geq 5$. Since $\Delta(G)=3$, it follows that $18=2 e(G)=\sum_{v \in V(G)} d(v) \leq x+3(9-x)=27-2 x \leq 17$, a contradiction. Furthermore, if $x=4$, then we claim that $y=1$. Assume, to the contrary, that $y \geq 2$. Then $18=2 e(G)=\sum_{v \in V(G)} d(v) \leq x+2 y+3(9-x-y)=27-2 x-y=19-y \leq 17$, a contradiction. Similarly, if $x=3$, then $y \leq 3$.

Suppose $x=4$ and $y=1$. Then $G$ is a unicyclic graph obtained by a cycle $C=$ $v_{1} v_{2} \ldots v_{5} v_{1}$ by adding four edges $v_{2} v_{6}, v_{3} v_{7}, v_{4} v_{8}, v_{5} v_{9}$. Choose $S=\left\{v_{6}, v_{7}, v_{9}\right\}$. Then $d_{G}(S) \geq 6$, which contradicts $\operatorname{sdiam}_{3}(G) \leq 5$. If $x=0$, then $G=C_{9}$, and hence $\operatorname{sdiam}_{3}(G)=6$ by Observation 2.1, a contradiction. If $x=1$, then $G$ is a unicyclic graph obtained by a cycle $C_{r}=v_{1} v_{2} \ldots v_{r} v_{1}$ and a path $P_{9-r}=w v_{r+1} \ldots v_{9}$ by identifying the vertex $v_{1}$ in $C_{r}$ and the endvertex $w$ in $P_{9-r}$. Choose $S=\left\{v_{\left\lfloor\frac{r}{3}\right\rfloor}, v_{\left\lfloor\frac{2 r}{3}\right\rfloor}, v_{9}\right\}$. Since $3 \leq r \leq 8$, it follows that $d_{G}(S) \geq\left\lfloor\frac{2 r}{3}\right\rfloor+(9-r) \geq 6$, which contradicts $\operatorname{sdiam}_{3}(G) \leq 5$.

Suppose $x=3$ and $y \leq 3$. Then $3 \leq r \leq 6$. Let $C_{r}(a, b, c)$ be a graph obtained from a cycle $C_{r}$ and three paths $P_{a}, P_{b}, P_{c}$ by adding three edges $z_{1} u_{a}, z_{2} u_{b}, z_{3} u_{c}$, where $0 \leq a \leq$ $b \leq c, 9=r+a+b+c-3, z_{1}, z_{2}, z_{3}$ are three distinct vertices in $C_{r}, u_{a}, u_{b}, u_{c}$ are leaves of $P_{a}, P_{b}, P_{c}$, respectively. If $r=3$, then $G=C_{3}(2,2,2)$ or $G=C_{3}(1,2,3)$ or $G=C_{3}(1,1,4)$. Choose $S$ consisting of all the three vertices of degree 1 in $G=C_{3}(2,2,2)$ or $G=C_{3}(1,2,3)$ or $G=C_{3}(1,1,4)$. Then $d_{G}(S) \geq 6$, which contradicts $\operatorname{sdiam}_{3}(G) \leq 5$. If $r=4$, then $G=C_{4}(1,2,2)$ or $G=C_{4}(1,1,3)$. Choose $S$ consisting of all the three vertices of degree 1 in $G=C_{4}(1,2,2)$ or $G=C_{4}(1,1,3)$. Then $d_{G}(S) \geq 6$, which contradicts $\operatorname{sdiam}_{3}(G) \leq 5$. If $r=5$, then $G=C_{5}(1,1,2)$. Choose $S$ consisting of all the three vertices of degree 1 in $G=C_{5}(1,1,2)$. Then $d_{G}(S) \geq 6$, which contradicts $\operatorname{sdiam}_{3}(G) \leq 5$. If $r=6$, then $G=C_{6}(1,1,1)$. One can easily check that $\operatorname{sdiam}_{3}(G) \geq 6$, a contradiction.

Suppose $x=2$. We define two graph classes as follows.

- Let $\mathcal{G}_{9}^{1}$ be a graph class, each graph $G$ of which is a unicyclic graph obtained by a cycle $C_{r}=z_{1} z_{2} \ldots z_{r} v_{1}$ and two paths $P_{s}=u_{1} u_{2} \ldots u_{s}, P_{t}=v_{1} v_{2} \ldots v_{t}$ by identifying a vertex $z_{i}$ of $C_{r}$ and the endvertex $u_{1}$ of $P_{s}$, and then identifying the other vertex $z_{j}$ of $C_{r}$ and the endvertex $v_{1}$ of $P_{t}$, where $9=r+s+t-2,3 \leq r \leq 7$ and $1 \leq i<j \leq r$.

- Let $\mathcal{G}_{9}^{2}$ be a graph class, each graph $G$ of which is a unicyclic graph obtained by a cycle $C_{r}=z_{1} z_{2} \ldots z_{r} z_{1}$ and $T_{a, b, c}$ (see Lemma 2.3) by identifying a vertex of $C_{r}$ and a leaf of $T_{a, b, c}$, where $9=r+a+b+c-3$ and $3 \leq r \leq 6$.

For $G \in \mathcal{G}_{9}^{1}, z_{i}$ and $z_{j}$ divide the cycle $C_{r}$ into two paths $Q_{r}^{1}=z_{i} z_{i+1} \ldots z_{j}$ and $Q_{r}^{2}=z_{j} z_{j+1} \ldots z_{r} \ldots z_{i}$. Without loss of generality, let $e\left(Q_{r}^{1}\right) \geq e\left(Q_{r}^{2}\right)$. Then $e\left(Q_{r}^{1}\right) \geq\left\lceil\frac{r}{2}\right\rceil$. Choose $S=\left\{u_{s}, v_{t}, z_{k}\right\}$ where $z_{k}$ is an internal vertex of $Q_{r}^{1}$. Since $3 \leq r \leq 7$, it follows 
that $d_{G}(S) \geq\left\lceil\frac{r}{2}\right\rceil+(9-r) \geq 6$, which contradicts $\operatorname{sdiam}_{3}(G) \leq 5$.

For $G \in \mathcal{G}_{9}^{2}$, let $u, v, w$ be the three leaves in $T_{a, b, c}$ and $w$ be the identifying vertex in $C_{r}$. Without loss of generality, let $w=v_{1}$. Choose $S=\left\{u, v, v_{\left\lfloor\frac{r}{2}\right\rfloor}\right\}$. Then $d_{G}(S) \geq$ $\left\lfloor\frac{r}{2}\right\rfloor+(9-r) \geq 6$, which contradicts $\operatorname{sdiam}_{3}(G) \leq 5$.

From Claim 1, we conclude that $e_{3}(9,3,5)=10=n+1$.

For $n=7$, let $G$ be a graph obtained from a cycle $C=v_{1} v_{2} \ldots v_{7}$ by adding three new edges $v_{1} v_{4}, v_{2} v_{5}, v_{3} v_{6}$. One can check that $\operatorname{sdiam}_{3}(G) \leq 3=n-4$ and hence this graph shows $e_{3}(7,3,3) \leq 10=n+3$. Similarly to the proof of $n=9$, we can prove that $e_{3}(7,3,3) \geq 10=n+3$. So $e_{3}(7,3,3)=10=n+3$. For $n=8$, let $G$ be a graph obtained from a cycle $C=v_{1} v_{2} \ldots v_{8}$ by adding two new edges $v_{1} v_{5}, v_{3} v_{7}$. One can check that $\operatorname{sdiam}_{3}(G) \leq 4=n-4$ and hence this graph shows $e_{3}(8,3,4) \leq 10=n+2$. Similarly to the proof of $n=9$, we can prove that $e_{3}(8,3,4) \geq 10=n+2$. So $e_{3}(8,3,4)=10=n+2$.

Lemma 4.6 For $n \geq 6$,

$$
e_{3}(n, 4, n-4)= \begin{cases}\left(\begin{array}{l}
n \\
2
\end{array}\right)-3 & \text { if } n=6 \\
n+2 & \text { if } n=7 \\
n-1 & \text { if } n \geq 8\end{cases}
$$

Proof. For $n \geq 8$, let $G=T(3, n-6,3)$. Then $G$ has exactly 6 leaves and $\Delta(G)=4$. From Lemma 2.1, $\operatorname{sdiam}_{3}(G) \leq n-4$. This tree shows that $e_{3}(n, 4, n-4) \leq n-1$ and hence $e_{3}(n, 4, n-4)=n-1$. For $n=7$, from (3) of Theorem $\left[3.2\right.$, we have $e_{3}(7,4,3)=9$. For $n=6$, let $G$ be a graph with $\Delta(G)=4$ and $\operatorname{sdiam}_{3}(G) \leq n-4=2$. From Lemma 2.4, we have $4 \leq \delta(G) \leq 5$, and hence $G$ is 4-regular. Clearly, $G$ is a graph obtained from $K_{6}$ by deleting a perfect matching. Therefore, $e(G)=\left(\begin{array}{l}6 \\ 2\end{array}\right)-3=12$ and $e_{3}(6,3,2)=12=\left(\begin{array}{l}n \\ 2\end{array}\right)-3$, as desired.

Lemma 4.7 For $n \geq 6$,

$$
e_{3}(n, 5, n-4)= \begin{cases}2 n+1 & \text { if } n=6 \\ n+2 & \text { if } n=7 \\ n-1 & \text { if } n \geq 8\end{cases}
$$

Proof. For $n \geq 8$, let $G=T(4, n-6,2)$. Then $G$ has exactly 6 leaves and $\Delta\left(T_{5}\right)=5$. From Lemma 2.1, $\operatorname{sdiam}_{3}(G) \leq n-4$. This tree shows that $e_{3}(n, 5, n-4) \leq n-1$ and hence $e_{3}(n, 5, n-4)=n-1$. For $n=7$, from (2) of Theorem [3.2, we have $e_{3}(7,5,3)=9$. For $n=6$, we let $G$ be the graph obtained from a complete graph $K_{6}$ by deleting a matching of size 2. It is clear that $\Delta(G)=5$ and $\operatorname{sdiam}_{3}(G)=2=n-4$. This graph shows that $e_{3}(6,5,2) \leq\left(\begin{array}{l}6 \\ 2\end{array}\right)-2=13$. We need to show that $e_{3}(6,5,2) \geq 13$. Let $G$ be a graph with $\Delta(G)=5$ and $\operatorname{sdiam}_{3}(G) \leq n-4=2$. From Lemma 2.4, we have $4 \leq \delta(G) \leq 5$. From 
this together with $\Delta(G)=5$ and $\operatorname{sdiam}_{3}(G)=2$, it follows that $e(G) \geq 13$ and hence $e_{3}(6,5,2) \geq 13$. So $e_{3}(6,5,2)=13$, as desired.

Theorem 4.3 (1) For $n \geq 5$,

$$
e_{3}(n, 2, n-4)= \begin{cases}\infty & \text { if } 5 \leq n \leq 9, \\ n & \text { if } n \geq 10\end{cases}
$$

(2) For $n \geq 6$,

$$
e_{3}(n, 3, n-4)= \begin{cases}\infty & \text { if } n=6, \\ n+3 & \text { if } n=7, \\ n+2 & \text { if } n=8, \\ n+1 & \text { if } n=9, \\ n-1 & \text { if } n \geq 10\end{cases}
$$

(3) For $n \geq 6$,

$$
e_{3}(n, 4, n-4)= \begin{cases}2 n & \text { if } n=6 \\ n+2 & \text { if } n=7 \\ n-1 & \text { if } n \geq 8\end{cases}
$$

(4) For $n \geq 6$,

$$
e_{3}(n, 5, n-4)= \begin{cases}2 n+1 & \text { if } n=6, \\ n+2 & \text { if } n=7 \\ n-1 & \text { if } n \geq 8\end{cases}
$$

(5) For $n \geq 7$ and $6 \leq \ell \leq n-1, e_{3}(n, \ell, n-4)=n-1$.

\section{For general $d$}

We now construct a graph and give an upper bound of $e_{3}(n, \ell, d)$ for general $\ell$ and $d$.

Proposition 5.1 For $4 \leq d \leq n-1$ and $2 \leq \ell \leq n-1$,

$$
e_{3}(n, \ell, d) \leq \frac{(n-d+1)(n-d+2)}{2}+d-3 .
$$

Proof. Let $U_{p}, W_{q}$ be two cliques of order $p, q$, respectively, where $p \geq q$ and $p+q=$ $n-d+1$. Set $V\left(U_{p}\right)=\left\{u_{1}, u_{2}, \ldots, u_{p}\right\}$ and $V\left(W_{q}\right)=\left\{w_{1}, w_{2}, \ldots, w_{q}\right\}$. Let $G$ be a graph defined as follow.

$$
\begin{aligned}
V(G)= & V\left(U_{p}\right) \cup V\left(W_{q}\right) \cup\left\{v_{i} \mid 1 \leq i \leq d-1\right\} \\
E(G)= & E\left(U_{p}\right) \cup E\left(W_{q}\right) \cup\left\{v_{1} u_{i}: 1 \leq i \leq p\right\} \cup\left\{v_{2} w_{i}: 1 \leq i \leq p\right\} \\
& \cup\left\{u_{i} w_{j}: 1 \leq i \leq p, 1 \leq j \leq q\right\} \cup\left\{v_{i} v_{i+1}: 2 \leq i \leq d-2\right\}
\end{aligned}
$$


It is clear that $|V(G)|=n, \Delta(G)=p+q=n-d+1$ and

$$
\begin{aligned}
|E(G)| & =\left(\begin{array}{l}
p \\
2
\end{array}\right)+\left(\begin{array}{l}
q \\
2
\end{array}\right)+p+q+p q+d-3 \\
& =\frac{(p+q)^{2}}{2}+\frac{p+q}{2}+d-3 \\
& =\frac{(n-d+1)(n-d+2)}{2}+d-3 .
\end{aligned}
$$

We need to show that $\operatorname{sdiam}_{3}(G) \leq d$. It suffices to prove that $d_{G}(S) \leq d$ for any $S \subseteq V(G)$ and $|S|=3$. Set $X=\left\{v_{1}, v_{2}, \ldots, v_{d-1}\right\}$. If $S \subseteq X$, the the tree induced by the edges in $\left\{v_{1} u_{1}, u_{1} w_{1}, u_{2} w_{1}\right\} \cup\left\{v_{i} v_{i+1} \mid 2 \leq i \leq d-2\right\}$ is an $S$-Steiner tree, and hence $d_{G}(S) \leq d$. If $|S \cap X|=2$, then $\left|S \cap V\left(U_{p}\right)\right|=1$ or $\left|S \cap V\left(W_{q}\right)\right|=1$. Without loss of generality, let $S \cap V\left(U_{p}\right)=\left\{u_{j}\right\}$. Then the tree induced by the edges in $\left\{v_{1} u_{j}, u_{j} w_{1}, u_{2} w_{1}\right\} \cup$ $\left\{v_{i} v_{i+1} \mid 2 \leq i \leq d-2\right\}$ is an $S$-Steiner tree, and hence $d_{G}(S) \leq d$. Suppose $|S \cap X|=1$. Then $\left|S \cap V\left(U_{p}\right)\right|=\left|S \cap V\left(W_{q}\right)\right|=1$ or $\left|S \cap V\left(U_{p}\right)\right|=2$ or $\left|S \cap V\left(W_{q}\right)\right|=2$. We first consider the case $\left|S \cap V\left(U_{p}\right)\right|=\left|S \cap V\left(W_{q}\right)\right|=1$. Without loss of generality, let $S \cap V\left(U_{p}\right)=\left\{u_{j}\right\}$ and $S \cap V\left(W_{q}\right)=\left\{w_{k}\right\}$. If $S=\left\{u_{j}, w_{k}, v_{1}\right\}$, then the tree induced by the edges in $\left\{u_{j} v_{1}, u_{j} w_{k}\right\}$ is an $S$-Steiner tree, and hence $d_{G}(S) \leq 2<d$. If $v_{1} \notin S$, then the tree induced by the edges in $\left\{u_{j} w_{k}, w_{k} u_{2}\right\} \cup\left\{v_{i} v_{i+1} \mid 2 \leq i \leq d-2\right\}$ is an $S$-Steiner tree, and hence $d_{G}(S)<d$. Next, we consider the case $\left|S \cap V\left(U_{p}\right)\right|=2$. Without loss of generality, let $S \cap V\left(U_{p}\right)=\left\{u_{j}, u_{k}\right\}$. If $S=\left\{u_{j}, u_{k}, v_{1}\right\}$, then induced by the edges in $\left\{u_{j} v_{1}, u_{k} v_{1}\right\}$ is an $S$-Steiner tree, and hence $d_{G}(S) \leq 2<d$. If $v_{1} \notin S$, then the tree induced by the edges in $\left\{u_{k} w_{1}, u_{j} w_{1}, w_{1} u_{2}\right\} \cup\left\{v_{i} v_{i+1} \mid 2 \leq i \leq d-2\right\}$ is an $S$-Steiner tree, and hence $d_{G}(S) \leq d$. In the end, we consider the case $\left|S \cap V\left(W_{q}\right)\right|=2$. Without loss of generality, let $S \cap V\left(W_{q}\right)=\left\{w_{j}, w_{k}\right\}$. If $S=\left\{u_{j}, u_{k}, v_{1}\right\}$, then induced by the edges in $\left\{w_{j} u_{1}, w_{k} u_{1}, v_{1} u_{1}\right\}$ is an $S$-Steiner tree, and hence $d_{G}(S) \leq 3<d$. If $v_{1} \notin S$, then the tree induced by the edges in $\left\{w_{k} v_{2}, w_{j} v_{2}\right\} \cup\left\{v_{i} v_{i+1} \mid 2 \leq i \leq d-2\right\}$ is an $S$-Steiner tree, and hence $d_{G}(S)<d$. We conclude that $e_{3}(n, \ell, d) \leq \frac{(n-d+1)(n-d+2)}{2}+d-3$.

\section{References}

[1] P. Ali, The Steiner diameter of a graph with prescribed girth, Discrete Math. 313(12) (2013), 1322-1326.

[2] P. Ali, P. Dankelmann, and S. Mukwembi, Upper bounds on the Steiner diameter of a graph, Discrete Appl. Math. 160(12) (2012), 1845-1850.

[3] P. Arunandhi, E. Cheng, C. Melekian, A note on the Steiner $k$-diameter of tensor product networks, Parall. Process. Lett. 29(2) (2019), 1950008. 
[4] G.S. Bloom, A characterization of graphs of diameter two, Amer. Math. Monthly 95(1) (1988), 37-38.

[5] B. Bollobás, Extremal Graph Theory, Acdemic press, 1978.

[6] B. Bollobás, Graphs with a given diameter and maximal valency and with a minimal number of edges, in: "Combinatorial Mathematics and its Applications" (Welsh, D.J.A., ed.) Academic Press London and New York, 1971), 25-37.

[7] J.A. Bondy and U.S.R. Murty, Graph Theory, GTM 244, Springer, 2008.

[8] F. Buckley, F. Harary, Distance in Graphs, Addision-Wesley, Redwood City, CA, 1990.

[9] J. Cáceresa, A. Márquezb, and M. L. Puertasa, Steiner distance and convexity in graphs, European J. Combin. 29(3) (2008), 726-736.

[10] G. Chartrand, O.R. Oellermann, S. Tian, and H.B. Zou, Steiner distance in graphs, Ćasopis pro pěstování matematiky 114 (1989), 399-410.

[11] G. Chartrand, F. Okamoto, and P. Zhang, Rainbow Trees in Graphs and Generalized Connectivity, Networks 55(4) (2010), 360-367.

[12] F.R.K. Chung, Diameter of graphs: Old problems and new results, 18th Southeastern Conf. on Combinatorics, Graph Theory and Computing, 1987.

[13] P. Dankelmann, H.C. Swart, and O.R. Oellermann, On the average Steiner distance of graphs with prescribed properties, Discrete Appl. Math. 79(1-3) (2008), 91-103.

[14] P. Dankelmann, H. Swart, O.R. Oellermann, Bounds on the Steiner diameter of a graph, Combinatorics, Graph Theory, and Algorithms, Vol. I, II, New Issues Press, Kalamazoo, MI, 1999.

[15] D.P. Day, O.R. Oellermann, and H.C. Swart, Steiner Distance-Hereditary Graphs, SIAM J. Discrete Math. 7(3) (1994), 437-442.

[16] D.Z. Du, Y.D. Lyuu, and D.F. Hsu, Line digraph iteration and connectivity analysis of de Bruijn and Kautz graphs, IEEE Trans. Comput. 42(5) (1994), 612-616.

[17] A. D'Atri and M. Moscarini, Distance-Hereditary Graphs, Steiner Trees, and Connected Domination, SIAM J. Comput. 17(3) (1988), 521-538.

[18] P. Erdös and A. Rényi, On a problem in the theory of graphs (in Hungarian), Publ. Math. Inst. Hungar. Acad. Sci. 7 (1962). 
[19] P. Erdös, A. Rényi, and V.T. Sós, On a problem of graph theory, Studia Sci. Math. Hungar. 1 (1966), 215-235.

[20] B. Furtula, I. Gutman, V. Katanić, Three-center Harary index and its applications, Iranian J. Math. Chem. 7(1) (2016), 61-68.

[21] C.E. Gillie, S.G. Kruk, E. Cheng, G. Wilson, Minimum Steiner tree for automatic SQL query generation applied on a medical record database, Proceedings of the Seventh IEEE 2011 World Congress on Services, (2011), 443-450.

[22] I. Gutman, On Steiner degree distance of trees, Appl. Math. Comput. 283 (2016), 163-167.

[23] I. Gutman, B. Furtula, X. Li, Multicenter Wiener indices and their applications, J. Serb. Chem. Soc. 80 (2015), 1009-1017.

[24] X. Li, Y. Mao, I. Gutman, The Steiner Wiener index of a graph, Discuss. Math. Graph Theory 36(2) (2016), 455-465.

[25] X. Li, Y. Mao, I. Gutman, Inverse problem on the Steiner Wiener index, Discuss. Math. Graph Theory 38 (2018), 83-95.

[26] F.J. Meyer, D.K. Pradhan, Flip trees, IEEE Trans. Computers 37(3) (1987), 472-478.

[27] M.R. Garey and D.S. Johnson, Computers and Intractibility: A Guide to the Theory of NP-Completeness, IEEE Trans. Computers, Freeman \& Company, New York, 1979.

[28] W. Goddard, O.R. Oellrmann, and H.C. Swart, Steiner distance stable graphs, Discrete Math. 132(1-3) (1994), 65-73.

[29] W. Goddard and O.R. Oellrmann, Distance in graphs, in: M. Dehmer (Ed.) Structural Analysis of Complex Networks, Birkhäuser, Dordrecht, 2011, 49-72.

[30] S.L. Hakimi, Steiner's problem in graph and its implications Networks 1(2) (1971), 113-133.

[31] N.P. Homenko, N.A. Ostroverhii, Diameter-critical graphs (in Russian), Ukrainian Math. J. 22 (1970), 637-646.

[32] N.P. Homenko, V.V. Strok, Some combinatorial indentities for sums of composition coefficient (in Russian), Ukrainian Math. J. 23 (1971), 830-837.

[33] F.K. Hwang, D.S. Richards, and P. Winter, The Steiner Tree Problem, NorthHolland, Amsterdam, 1992. 
[34] D.F. Hsu, On container width and length in graphs, groups, and networks, IEICE Transaction on Fundamentals of Electronics, Communications and Computer Science, E77-A (1994), 668-680.

[35] D.F. Hsu and T. Euczak, Note on the $k$-diameter of $k$-regular $k$-connected graphs, Discrete Math. 133(1-3) (1994), 291-296.

[36] A.Y. Levi, Algorithm for shortest connection of a group of graph vertices, Sov. Math. Dokl. 12 (1971), 1477-1481.

[37] Y. Mao, The Steiner diameter of a graph, Bull. Iran. Math. Soc. 43(2)(2017), 439-454.

[38] Y. Mao, E. Cheng, Z. Wang, Steiner distance in product networks, Discrete Math. Theor. Comput. Sci. 20(2) (2018), \#8.

[39] Y. Mao, K.C. Das, Steiner Gutman index, MATCH Commun. Math. Comput. Chem. 79 (2018), 779-794.

[40] Y. Mao, Z. Wang, I. Gutman, A. Klobučar, Steiner degree distance, MATCH Commun. Math. Comput. Chem. 78(1) (2017), 221-230.

[41] Y. Mao, Z. Wang, I. Gutman, H. Li, Nordhaus-Gaddum-type results for the Steiner Wiener index of graphs, Discrete Appl. Math. 219 (2017), 167-175.

[42] F.J. Meyer, D.K. Pradhan, Flip trees, IEEE Trans. Comput. 37(3) (1987), 472-478.

[43] O. Ore, Diameter in graphs, J. Combin. Theory 5 (1968), 75-81.

[44] N. Tratnik, On the Steiner hyper-Wiener index of a graph, Appl. Math. Comput. 337(15) (2019), 360-371.

[45] M.E. Watkins, A lower bound for the number of vertices of a graph, Amer. Math. Monthly 74 (1976), 297. 